\title{
The Condition Order Spectrum
}

\author{
Boushra Y. Hussein \\ Department of Mathematics \\ College of Education \\ University of AL-Qadisiyah \\ Boushra.alshebani@qu.edu.iq
}

\author{
Sara F. Hassan \\ Department of Mathematics \\ College of Computer Science \\ and Information Technology \\ University of AL-Qadisiyah \\ Sarafahim66@yahoo.com
}

Recived : $2418 \backslash 2017$

Revised : $14 \backslash 9 \backslash 2017$

Accepted : $2019 \backslash 2017$

\begin{abstract}
In this paper, we will study the relation between complex character $f$ and the condition order spectrum $\mathcal{S P}_{\mathcal{E}}(a)$ in real and complex ordered Banach algebra $A$.We prove many properties on it's . afterwards, we prove that $\delta \mathcal{P}_{\varepsilon}(a)$ is upper semi continuous Finally we will show the relation between condition Quasinilpotent $\mathcal{Q} \mathcal{N}_{\varepsilon}(A)$ and the radical .
\end{abstract}

\section{Mathematical subject classification: 46A40, 46B04, 46E25, 47C05}

Keywords: Spectrum Order Banach algebra, Strong algebra cone in $O B A$, condition Quasi nilpotent, the radical and The condition pseudospectrum.

\section{Introduction and Preliminaries .}

The study of spectrum has been started and developed turn of the century in[G. Frobenius, 1908] for the canonical order of $\mathbb{R}^{n}$, [Krien-Rutman,1939] for Banach space, [Helmut H. Schaefer, 1986] for order topological linear space, [H. Raubenheimer and S. Rode,1996] for ordered Banach algebra and [E. B. Daies introduce( Spectral Theory and Differential Operators) ,1996] , [K. Yosida introduce (On the Theory of Spectra),1940].

in [D. Sukumar 2007] introduce the concept of condition spectrum in real and complex Banach algebra. In this chapter we introduce four sections

Section one, focuses upon the introduction and Preliminaries of the paper

Section two, concentrates on a new concept of a spectrum of elements in real and complex unital order Banach algebra is the condition order spectrum and condition order radius. We will prove some properties different from the properties to the usual spectrum which is a subset of condition order spectrum.

Section three, involves the properties of condition order spectrum and the concept of the condition of Quisinilpotent. This section will prove some of the properties to shows the relation between Quisinilpotent and Radical. Section four, shown the relation between character and condition order spectrum. Also, we will introduce the concept of pseudo spectrum in a condition different from condition spectrum in [4].

Definitions 1.1[5]:

- A partially ordered set $(A, \geqslant)$ is called direct set if for all $\alpha, \beta$ in $A$ such that $\alpha \neq \beta$,then there exists $\mu$ such that $\mu \geqslant \alpha, \mu \geqslant \beta$.

- $A$ function $f$ from a direct set $A$ to a nonempty set $X$ is called a net on $X$ and it is denoted by $\left\{f_{\lambda}\right\}_{\lambda \in \Lambda}$. 
Definition 1.2[6]: Let $A$ be a real or complex Banach algebra with identity and $C$ non-empty subset of $A$.We call $C$ a cone if it satisfies the following

1. $a+b \in C \quad$ for all $a, b \in C$

2. $\beta a \in C$ for all $a \in C$ and $\beta \geq 0$.

In addition, if $C$ satisfies $C \cap-C=\{0\}$, then

$C$ will be called a proper cone induced an ordering $(\preccurlyeq)$ on $A$ by $a \preccurlyeq b$,if and only if $b-a \epsilon C$ for all $a, b \in A$ We say that $C$ is algebra cone if it is satisfies the following:

1. $a \cdot b \in C$ for all $a, b \in C$,

2. $e \in C$.

Definition 1.3(Ordered Banach Algebras)[6]: Every complex Banach algebra $A$ with identity generated by $C$ is called ordered Banach algebra $(O B A)$ if $\mathrm{A}$ is ordered by a relation $(\preccurlyeq)$ such that for every $n, m, c \in A$ and $\beta \geq 0$

$a, b \geqslant 0 \Rightarrow a+b \geqslant 0$

1. $a \geqslant 0, \lambda \geq 0 \Longrightarrow \lambda a \geqslant 0$

2. $a, b \geqslant 0 \Rightarrow a . b \geqslant 0$.

so if $A$ is ordered by an algebra cone $C$, we will obtain $(A, C)$ which is an ordered Banach algebra.

Example 1.4[7]: Let $A$ be Banach algebra and $C=\{\alpha e: \alpha \geq 0\}$ then $C$ is closed, algebra cone of $A$, then $(A, C)$ be an ordered Banach algebra.

Example 1.5 [7]: Let $\mathbb{C}$ be Banach algebra of all complex numbers with norm $\|x\|=$ $|x|$ and the subset of $\mathbb{C}$ of all non-negative real numbers $\mathbb{R}^{+}$. Then $\mathbb{R}^{+}$is a closed, algebra cone of $\mathbb{C}$ and $\left(\mathbb{C}, \mathbb{R}^{+}\right)$be an order Banach algebra.
Definition 1.6(Character)[8]: Let $A$ be

Banach algebra for all $n \epsilon A$ and $\beta \epsilon F$, we call the functional $f$ is Character if it satisfies:

i- Linear

that is $f(\mathfrak{a}+b)=f(\mathfrak{a})+f(b)$

and $\beta f(a)=f(\beta a)$

ii- Multiplicative

that is $f(a b)=f(a) f(b)$

iii- $f(e)=1$

If $F=\mathbb{R}$, we say that $f$ is real character, and $\mathcal{M}_{A}$ denote the set of all real character by If $F=\mathbb{C}$, we say that $f$ is complex character, $\operatorname{Ch}(A)$ denotes the set of all complex character.

Theorem 1.7(Gelfand-Mazur)[5]: Let $A$ is Banach algebra which is every non-zero element is invertible, and then there is a unique isomorphism from $A$ into $\mathbb{C}$.

Theorem 1.7(Automatic Continuity of

Character)[5]: let $A$ be a Banach algebra over $F$ and let $f: A \rightarrow F$ be a character then $f$ is continuous and has the norm $\|f\| \leq 1$.

2 Condition order spectrum properties order in Banach algebra

Definition 2.1: Let $A$ be $O B A$ with algebra cone $C$, we say that $C$ is invertible algebra cone if every element in $C$ has inverse. We denote that the set of all invertible elements in $A$ by $I N(C)$. 
Definition 2.2 (Spectrum): Let $A$ be $O B A$ and $a \in A$, the order spectrum of $a$ which denoted by $\mathcal{S P}(a)$ is defined by:

$\mathcal{S P}(a)=\{\lambda \in \mathbb{C}: \lambda-a \notin I N(C)\}$

Definition 2.3: Let $A$ be $O B A$ and $a \in A$, the order spectrum radius of $a$ which denoted by $r(a)$ is defined by :

$r(a)=\sup \{|\lambda|: \lambda \in \mathcal{S P}(a)\}$.

Proposition 2.4: Let $(A, C)$ be $O B A$.If $C$ is inverse-closed then $\quad \mathcal{S P}(a, A)=\mathcal{S P}(a, C)$ for $a \in A$.

Proof:-Since $C \subseteq A$ is inverse - closed so

$I N(c) \subseteq I N(a)$

let $\lambda \in \mathcal{S P}(a, A)$. Then $\lambda-a \notin I N(a)$ therefore $\lambda-a \notin I N(c)$ and $\lambda \in \mathcal{S P}(a, C)$.

Conversely, let $\lambda \in \mathcal{S P}(a, C)$ then $\lambda-a \notin I N(c)$

If $\lambda-a \in I N(a)$ so $\lambda-a$ has inverse, since $C$ is inverse -closed then $\lambda-a \epsilon I N(c)$ this contradiction

$\square$

Definition2.5 (Spectrum Order Banach algebra) : Let $A$ be $O B A$ with identity and $a \in A$, the spectrum which denoted by $\mathcal{S} \mathcal{P}_{o}(a)$ is define by

$\delta \mathcal{P}_{o}(a)=\left\{\alpha+i t \in \mathbb{C}:(\alpha-a)^{2}+t^{2} \notin I N(C)\right\}$.

The complexification of a real order Banach algebra $A$ denoted by $A_{\mathbb{C}}$ such that $A_{\mathbb{C}}=A \times A$,

Let $(a, b),(c, d) \in A_{\mathbb{C}}$ and $\alpha+i \beta \in \mathbb{C}$

$(a, b)+(c, d)=(a+c, b+d)$

$\alpha+i \beta(a, b)=(\alpha a-\beta b, \alpha b+\beta a)$

$(a, b),(c, d)=(a c-b d, a d+b c)$.

With these operations $A_{\mathbb{C}}$ become complex order Banach algebra, if $A$ has identity $e$, then $(e, 0)$ becomes the identity of $A_{\mathbb{C}}$.

And the complexification to $C$ is $C_{\mathbb{C}}=C \times C$.
Definition 2.6(Strong algebra cone in

OBA):Let $A$ be a $O B A$ with identity, we say that $C$ is Strong algebra cone in $O B A$ if it satisfies the following:

- If $C$ is an invertible algebra cone .

- $\lambda a \epsilon C$ for all $a \epsilon C$ and $\lambda \epsilon F^{*}=F \backslash\{0\}$

Definition 2.7: Let $0<\varepsilon<1$, define

$C_{\varepsilon}=\left\{a \in I N(a):\|a\|\left\|a^{-1}\right\|<\frac{1}{\varepsilon}\right\}$

In complexification,

$$
\begin{aligned}
& C_{\varepsilon}^{\prime}=\left\{(a, 0) \epsilon I N((a, 0)): \|((\alpha, 0)-(a, 0))^{2}+\right. \\
& \left.\left.t^{2}\|\|((\alpha, 0)-(a, 0))^{2}+t^{2}\right)^{-1} \|<\frac{1}{\varepsilon}\right\}
\end{aligned}
$$

Definition 2.8 (Condition order spectrum): Let $A$ be a complex $O B A$ and $a \epsilon A$ with $0<\varepsilon<1$ the condition order spectrum of $a$ for $\varepsilon$ is defined by $\mathcal{S} \mathcal{P}_{\varepsilon}(a)=\left\{\lambda \in \mathbb{C}:\|\lambda-a\|\left\|(\lambda-a)^{-1}\right\| \geq \frac{1}{\varepsilon}\right\}$

Remark 2.9: $\delta \mathcal{P}_{\mathcal{\varepsilon}}\left(a^{-1}\right)=\left\{\lambda^{-1}: \lambda \in \mathcal{S} \mathcal{P}_{\varepsilon}(a)\right\}$

If $A$ be a real $O B A$ the condition order spectrum is defined by $\delta \mathcal{P}_{\mathcal{\varepsilon}}(a)=\left\{\alpha+i t \in \mathbb{C}:(\alpha-a)^{2}+\right.$ $\left.t^{2} \notin C_{\varepsilon}\right\}$

$=\left\{\alpha+i t \in \mathbb{C}:\left\|(\alpha-a)^{2}+t^{2}\right\| \|\left((\alpha-a)^{2}+\right.\right.$ $\left.\left.t^{2}\right)^{-1} \| \geq \frac{1}{\varepsilon}\right\}$.

In complexification,

$$
\begin{aligned}
& \mathcal{S P}_{\varepsilon}((a, 0))=\left\{\alpha+i t \in \mathbb{C}: \|((\alpha, 0)-(a, 0))^{2}+\right. \\
& \left.\left.t^{2}\|\|((\alpha, 0)-(a, 0))^{2}+t^{2}\right)^{-1} \| \notin C_{\varepsilon}^{\prime}\right\} \\
& =\left\{\alpha+i t \in \mathbb{C}: \|((\alpha, 0)-(a, 0))^{2}+\right. \\
& \left.\left.t^{2}\|\|((\alpha, 0)-(a, 0))^{2}+t^{2}\right)^{-1} \| \geq \frac{1}{\varepsilon}\right\}
\end{aligned}
$$

The condition order spectral radius which denoted by $r_{\varepsilon}(a)$ is define by $r_{\varepsilon}(a)=$ $\sup \left\{|\lambda|: \lambda \in \mathcal{S P}_{\varepsilon}(a)\right\}$.

$$
\text { and } r_{\varepsilon}\left(a^{-1}\right)=\sup \left\{\left|\lambda^{-1}\right|: \lambda \in \mathcal{S} \mathcal{P}_{\varepsilon}\left(a^{-1}\right)\right\}
$$




\section{Proposition 2.10:}

1. $a \in I N(C)$ if and only if $(a, 0) \in I N\left(C_{\mathbb{C}}\right)$.

2. $(a, b) \in I N(C)$ if and only if $(a,-b) \in I N\left(C_{\mathbb{C}}\right)$.

Proof:- It is clear from definition.

Proposition $2.11: \delta \mathcal{P}_{\varepsilon}\left((a, 0), A_{\mathbb{C}}\right)=\delta \mathcal{P}_{\varepsilon}(a)$

Proof:-Let $\alpha+i t \in \mathcal{S P}_{\varepsilon}(a)$

Then there is $a \in I N\left(C_{\mathbb{C}}\right)$ such that

$\left\|(\alpha-a)^{2}+t^{2}\right\|\left\|(\alpha-a)^{2}+t^{2}\right\|^{-1} \geq \frac{1}{\varepsilon}$

then by proposition(4.2.10(1))

$\left\|((\alpha, 0)-(a, 0))^{2}+t^{2}\right\| \|((\alpha, 0)-(a, 0))^{2}+$ $t^{2} \|^{-1} \geq \frac{1}{\varepsilon}$

then $(\alpha-a, t)(\alpha-a,-t) \notin C_{\varepsilon}^{\prime}$

and by proposition $(4.2 .10(2))(\alpha-a, t) \notin C_{\varepsilon}^{\prime}$

then $(\alpha+i t)(1,0)-(a, 0) \notin C_{\varepsilon}^{\prime}$

then $\alpha+i t \in \mathcal{S} \mathcal{P}_{\varepsilon}\left((a, 0), A_{\mathbb{C}}\right)$.

Theorem 2.12: $\mathcal{S P}(a) \subseteq \mathcal{S} \mathcal{P}_{\mathcal{\varepsilon}}(a)$.

Proof:-Let $\lambda \in \mathcal{S P}(a)$ then $\lambda-a \notin I N(C)$ so $\lambda-a \notin C_{\varepsilon}$.

Then $\|\lambda-a\|\|\lambda-\alpha\|^{-1} \geq \frac{1}{\varepsilon}$, so $\lambda \in \mathcal{S} \mathcal{P}_{\varepsilon}(a)$.

Proposition 2.13: Let $(A, C)$ with algebra cone $\mathrm{C}$ and $B$ be subalgebra of $A$ with algebra cone $C \cap$ $B$ and $e \in B \subset A$ such that the condition spectrum radius in $B$ is monotone. If $a, b \in A$ such that $o \leq a \leq b$ relative to $C$ and $r_{\varepsilon}^{B}(b)=r_{\varepsilon}^{A}(b)$ then $r_{\varepsilon}^{A}(a) \leq r_{\varepsilon}^{A}(b)$.

Proof:-Since $B$ is subalgebra of $A$, then $(B, C \cap$ $B$ )is ordered Banach algebra by complex (theorem 2.3.8[9]), the condition spectrum radius is monotone then $r_{\varepsilon}{ }^{B}(a) \leq r_{\varepsilon}{ }^{B}(b)$, since $B \subseteq A$ and $B$ is subalgebra, then $\delta \mathcal{P}_{\mathcal{\varepsilon}}{ }^{A}(a) \subseteq \delta \mathcal{P}_{\mathcal{\varepsilon}}{ }^{B}(a)$ so $r_{\varepsilon}^{A}(a) \leq r_{\varepsilon}^{B}(a)$ and we have $r_{\varepsilon}^{A}(b)=$ $r_{\varepsilon}^{B}(b)$, then we obtain $r_{\varepsilon}^{A}(a) \leq r_{\varepsilon}^{B}(a) \leq r_{\varepsilon}^{B}(b)=r_{\varepsilon}^{A}(b)$

$\square$

Theorem 2.14: $r(a) \leq r_{\varepsilon}(a) \leq \frac{1+\varepsilon}{1-\varepsilon}\|a\|$

Proof:-Since $\mathcal{S P}(a) \subseteq \mathcal{S} \mathcal{P}_{\mathcal{\varepsilon}}(a)$, then $r(a) \leq$ $r_{\varepsilon}(a)$

Let $\alpha+i t \in \mathbb{C} \quad a \in A$ If $|\alpha+i t| \leq\|a\|$ since $(1-\varepsilon)\|a\| \leq(1+$

$\varepsilon)\|a\|$, then $|\alpha+i t| \leq \frac{1+\varepsilon}{1-\varepsilon}\|a\|$.

If $|\alpha+i t| \geq\|a\|$, then $(\alpha-a)^{2}+t^{2}$ is invertible by

proposition(2.2.22) and $\left\|\left((\alpha-a)^{2}+t^{2}\right)^{-1}\right\| \leq$ $\frac{1}{|\alpha+i t|-\|a\|}$

Then $\left\|(\alpha-a)^{2}+t^{2}\right\|\left\|\left((\alpha-a)^{2}+t^{2}\right)^{-1}\right\| \geq$ $\frac{1}{\varepsilon} \quad$ so $\frac{|\alpha+i t|+\|a\|}{|\alpha+i t|-\|a\|} \geq \frac{1}{\varepsilon}$

and $\frac{\varepsilon|\alpha+i t|+\|a\|}{|\alpha+i t|-\|a\|} \geq 1$

$\varepsilon(|\alpha+i t|+\|a\| \geq|\alpha+i t|-\|a\|$

$\varepsilon|\alpha+i t|+\varepsilon\|a\| \geq|\alpha+i t|-\|a\|$

$\|a\|+\varepsilon\|a\| \geq|\alpha+i t|-\varepsilon|\alpha+i t|$

$(1+\varepsilon)\|a\| \geq(1-\varepsilon)|\alpha+i t|$

$|\alpha+i t| \leq \frac{1+\varepsilon}{1-\varepsilon}\|a\|$.

In [2], we have the next example which explains that the radius is not a necessary condition radius and the spectrum is not a necessary condition spectrum. 
Example(Bilateral Shift) 2.15: Let $f: \ell^{2}(\mathbb{Z}) \rightarrow$ $\ell^{2}(\mathbb{Z})$ define by $f\left(e_{i}\right)=e_{i+1}$ for all $i \in \mathbb{Z}$, such that $e_{i}$ is the standard basis. Let $\beta \in \mathbb{C}$, consider $f-\beta$ define by $b(t)=-\beta+e^{i t}$

Such that $\|f-\beta\|=\|b(t)\|_{\infty}$. Hence $\|f-\beta\|=|\beta|+1$. When $\beta \neq 1$ we have $\beta \notin \mathcal{S P}(a)$ and $(f-\beta)^{-1}=\frac{1}{b(t)}$, then

$\left\|(f-\beta)^{-1}\right\|=\frac{1}{\|b(t)\|_{\infty}}=\frac{1}{\inf 0 \leq t \leq 2 \pi|b(t)|}$ $= \begin{cases}\frac{1}{|\beta|-1} & \text { if }|\beta|>1 \\ \frac{1}{1-|\beta|} & \text { if }|\beta|<1\end{cases}$

So, $\|(f-\beta)\|\left\|(f-\beta)^{-1}\right\|=$ $\left\{\begin{array}{l}\frac{|\beta|+1}{|\beta|-1} \text { if }|\beta|>1 \\ \frac{1+|\beta|}{1-|\beta|} \text { if }|\beta|<1\end{array}\right.$

From this $\|(f-\beta)\|\left\|(f-\beta)^{-1}\right\| \geq \frac{1}{\varepsilon}$ if and only if $1>|\beta| \geq \frac{1-\varepsilon}{1+\varepsilon}$ or $\frac{1+\varepsilon}{1-\varepsilon} \geq|\beta|>1$. It is known that $\mathcal{S P}(f)=\{\beta:|\beta|=1\}$ which is the Range of $f$. Hence $\mathcal{S} \mathcal{P}_{\varepsilon}(a)=\left\{\beta: \frac{1-\varepsilon}{1+\varepsilon} \leq|\beta| \leq\right.$ $\left.\frac{1+\varepsilon}{1-\varepsilon}\right\}$.

Theorem 2.15: Let $A$ be a real $O B A$ with identity and let $f$ be a complex character from $A$ to $\mathbb{C}$ with $f(e)=1$, then $f(a) \in \mathcal{S} \mathcal{P}_{\varepsilon}(a)$ for all $a \in A$.

Proof :- Let $a \in A$ and $f(a)=\alpha+i t$ and $(\alpha-a)^{2}+t^{2}$ is invertible, then $1=|f(e)|=\mid f\left((\alpha-a)^{2}+t^{2}\right)\left((\alpha-a)^{2}+\right.$ $\left.t^{2}\right)^{-1} \mid \leq \varepsilon\left\|(\alpha-a)^{2}+t^{2}\right\| \|(\alpha-a)^{2}+$ $\left.t^{2}\right)^{-1} \|$ so $\left.\left\|(\alpha-a)^{2}+t^{2}\right\| \|(\alpha-a)^{2}+t^{2}\right)^{-1} \| \geq \frac{1}{\varepsilon}$ Then $f(a)=\alpha+i t \in \mathcal{S} \mathcal{P}_{\varepsilon}(a)$.
Theorem 2.16: Let $A$ be a real $O B A$ with identity, let $0<\varepsilon<1$ and $f$ be a linear functional from $A$ to $\mathbb{C}$.If $f(a) \in \mathcal{S} \mathcal{P}_{\varepsilon}(a)$, then $f$ is character.

Proof :-Since $S \mathcal{P}_{\varepsilon}(e)=\{1\}$, then $f(e)=1$

let $a \in A$ and $\|a\|=1$, by theorem(4.2.14) we have $\|f\| \leq \frac{1+\varepsilon}{1-\varepsilon}\|a\|$

define $\varphi: \mathbb{C} \rightarrow \mathbb{C}$ by $\varphi(\alpha+i t)=f(\exp (\alpha+$ it) (a)

then $\varphi$ is entire function since

$f(a) \in \mathcal{S} \mathcal{P}_{\varepsilon}(a), \varphi(\alpha+i t) \neq 0$

we have that $|\varphi(\alpha+i t)| \leq\|f\| \| \exp (\mid \alpha+$ $i t \mid a) \| \leq \frac{1+\varepsilon}{1-\varepsilon} \exp (|\alpha+i t| .1)$.

We can write $\varphi(\alpha+i t)$ by the way: $\varphi(\alpha+$ $i t)=\exp (g(\alpha+i t))$ for some Entire function $g$ which define by $g(\alpha+i t)=\delta(\alpha+i t)+\beta$ by [37] since $f(\exp (0))=1, \varphi(\exp (0))=1$ and $\varphi(0)=\exp (g(\alpha+i t))=1$ since $1=$ $\exp (0)$, then $f(a)(\alpha+i t)+\beta=0$, so $\beta=0$ and

$$
\begin{aligned}
& g(\alpha+i t)=f(a)(\alpha+i t), \text { we obtain } \\
& \varphi(\alpha+i t)=\exp (f(a)(\alpha+i t)) \\
& =\sum_{i=0}^{n} \frac{f(a)^{n}}{n !}(\alpha+i t)^{n}
\end{aligned}
$$

by(1.1) we have

$\varphi(\alpha+i t)=f\left[\sum_{i=0}^{n} \frac{a^{n}}{n !}(\alpha+i t)^{n}\right]=$ $\sum_{i=0}^{n} \frac{f\left(a^{n}\right)}{n !}(\alpha+i t)^{n}$ by comparing with (1.2) $f\left(a^{n}\right)=f(a)^{n}$.

3 further properties of condition order spectrum 
Definition 3.1(condition Quasinilpotent) : Let $A$ order Banach algebra. Then $A$ is called condition Quasi nilpotent if $\mathcal{S} \mathcal{P}_{\varepsilon}(a)=\{0\}$ and denoted by $Q \mathcal{N}_{\varepsilon}(A)$.

Definition 3.2 (Radical ): Let $A$ be $O B A$ with identity , then the radical which denoted by $\operatorname{Rad}(A)$ is define as $\operatorname{Rad}(A)=\{x \in A: e-$ $x z \in C: z \in A\}$

Proposition3.3: $\operatorname{Rad}(A)=\{a \in A: a A \subset$ $\left.\mathcal{Q} \mathcal{N}_{\varepsilon}(A)\right\}=\left[a \epsilon A: A a \subset Q \mathcal{N}_{\varepsilon}(A)\right\}$

Proof:-Let $a \in\left\{a \in A: a A \subset Q \mathcal{N}_{\varepsilon}(A)\right\}$. Then $\mathcal{S P}_{\mathcal{\varepsilon}}(a z)=\{0\}$

For all $z \epsilon A$, so $1-z a \epsilon C$ which implies that $a \in \operatorname{Rad}(A)$ conversely, let $a \in \operatorname{Rad}(A)$. Then $1-z a \in C$ for all $z \in A$ this implies $1-z a \epsilon C$ is invertible, so $\mathcal{S} \mathcal{P}_{\mathcal{\varepsilon}}(a z)=\{0\}$ for all $z \in A$ we obtain $a z \in Q \mathcal{N}_{\varepsilon}(A)$, then $a A \subset Q \mathcal{Q} \mathcal{N}_{\varepsilon}(A)$.

口

Corollary 3.4: $\operatorname{Rad}(A) \subset Q \mathcal{N}_{\varepsilon}(A)$.

Proof:-It is clear .

Proposition 3.5: Let $(A, C)$ be a $O B A$ and the condition spectral radius is monotone. Then the following are satisfies:

1. If $b$ is Quasi nilpotent, then $a$ is Quasi nilpotent

2. If $b \in \operatorname{Rad}(A)$, then $a$ is Quasi nilpotent

3. If $b \in \operatorname{Rad}(A)$ and $a$ in the center of $A$ then $a \in \operatorname{Rad}(A)$.

\section{Proof:-}

1. Since $b \in Q \mathcal{Q} \mathcal{N}_{\varepsilon}(A)$ then $r_{\varepsilon}(b)=0$, since $0 \leq r_{\varepsilon}(a) \leq 0$ then we give $\mathcal{S} \mathcal{P}_{\varepsilon}(a)=\{0\}$.

2. Since $b \in \operatorname{Rad}(A)$, then $b \in Q \mathcal{Q} \mathcal{N}_{\varepsilon}(A)$ by corollary(4.3.4) so by (1) above we have $a \in Q \mathcal{N}_{\varepsilon}(A)$.
3. Since $b \in \operatorname{Rad}(A)$ then $b \in Q \mathcal{N}_{\varepsilon}(A)$ so $r_{\varepsilon}(a)=0$ let $x \in A$, since $x a=a x$ then $r_{\varepsilon}(a x) \leq$ $r_{\varepsilon}(a) r_{\varepsilon}(x) \leq 0 . r_{\varepsilon}(x)=0$ we obtain $a A \subseteq$ $Q \mathcal{N}_{\varepsilon}(A)$, then $\operatorname{a\epsilon } \operatorname{Rad}(A)$.

$\square$

Theorem 3.6: Let $A$ be $O B A$ and the condition spectrum radius is monotone such that $0 \leq a \leq$ $b$.If $b \in \operatorname{Rad}(A)$ then $a C \subset Q \mathcal{N}_{\varepsilon}(A)$.

Proof:- Let $b \in \operatorname{Rad}(A)$. Then $b c \subset \operatorname{Rad}(A) \subset$ $Q \mathcal{N}_{\varepsilon}(A)$ so $r_{\varepsilon}(b c)=0$, since $o \leq a \leq b$, then $0 \leq a c \leq b c$ for all $c \in C$ and $r_{\varepsilon}(a c) \leq r_{\varepsilon}(b c)$ because of the condition spectrum radius is monotone we obtain that $r_{\varepsilon}(a c)=0$ then $a C \subset Q \mathcal{N}_{\varepsilon}(A)$.

$\square$

\section{The Relation Between Character And Condition Order Spectrum}

Theorem 4.1: Let $A$ be $O B A$ and $a \epsilon A$ then $\mathcal{S P}_{\mathcal{E}}(a)$ is upper semi continuous on $A$, that is: If $a \epsilon A$ then for every open set $U$ containing $\mathcal{S P}_{\mathcal{\varepsilon}}(a)$ there exists $\delta>0$ such that $\|a-b\|<$ $\delta$ implies $\quad \delta \mathcal{P}_{\varepsilon}(b) \subset U$

Proof:-Suppose that there exists sequence $\left\{b_{n}\right\}$ and $\left\{\alpha_{n}\right\}$ such that $a=\lim _{n \rightarrow \infty} b_{n}, \alpha_{n} \in \mathcal{S} \mathcal{P}_{\varepsilon}\left(b_{n}\right) \cap U^{c}$ from theorem (4.2.14[9]). Then $\left|\alpha_{n}\right| \leq \frac{1+\varepsilon}{1-\varepsilon}\left\|b_{n}\right\|$ so $\left\{\alpha_{n}\right\}$ is bounded sequence in $\mathbb{C}$ and therefore has a convergent subsequence .(without loss a generality ) we may assume that $\left\{\alpha_{n}\right\}$ convergent say to $\alpha$ that is $\left(\alpha_{n}-b_{n}\right)^{2}+t^{2} \rightarrow(\alpha-a)^{2}+$ $t^{2}$ as $n \rightarrow \infty$, since $U^{c}$ is closed, so $\bar{U}=U^{c}$ then $\alpha \notin U$ we obtain that $\alpha-a$ is invertible ,since the set of all invertible is open so for $n$ large enough $\alpha_{n}-b_{n}$ is invertible which is contradiction. 
Theorem 4.2: Let $A$ be $O B A$ and $a \in A$, let $U, V$ are two disjoint open set such that $\mathcal{S P}_{\varepsilon}(a) \subset$ $U \cup V$ and $\mathcal{S} \mathcal{P}_{\varepsilon}(a) \cap U \neq 0$. then there exists $r>0$ such that $\|a-b\|<r$ implies $\mathcal{S} \mathcal{P}_{\varepsilon}(b)$ $\cap U \neq 0$

Proof:- Since the spectrum is upper semi continuous there exists $\delta>0$ such that $\| a-$ $b \|<\delta$ imply $\delta \mathcal{P}_{\varepsilon}(b) \subset U U V$

Suppose that there exists a sequence $b_{n} \rightarrow a$ such that $\delta \mathcal{P}_{\varepsilon}(b) \subset V$ for $n$ large enough let $f$ be a function on $U \cup V$ define by $f(a)=1$ if $a \in U$ and $f(a)=0$ if $a \in \mathcal{V}$, then $f$ is holomorphic on $U \cup V$, since $b_{n} \rightarrow a$ and the definition of the functional culculas we see that $\lim _{n \rightarrow \infty} f\left(b_{n}\right)=$ $f(a)$ and $f\left(b_{n}\right)=0$ for $n$ large enough

$$
\text { , since } \mathcal{S P}(f(a)) \subseteq \mathcal{S} \mathcal{P}_{\varepsilon}(f(a))=
$$

$\delta \mathcal{P}_{\mathcal{E}}(0)=\{0\}$, then $\mathcal{S P}(f(a)) \subseteq\{0\}$

since $\mathcal{S P}(f(a))=f(\mathcal{S P}(a)) \quad$ by spectral mapping theory [1], then $f(\mathcal{S P}(a)) \subseteq\{0\}$ but $f(\mathcal{S P}(a)) \subseteq f\left(\mathcal{S P}_{\varepsilon}(a)\right)$, then $f\left(\mathcal{S P}_{\mathcal{\varepsilon}}(a)\right)=\{0\}$ so we obtain $f\left(\mathcal{S \mathcal { P } _ { \varepsilon }}(a)\right)=\delta \mathcal{P}_{\varepsilon}(f(a))=\{0\}$ but $\mathcal{S} \mathcal{P}_{\varepsilon}(f(a))$ contain 1 and this contradiction Then $\mathcal{S} \mathcal{P}_{\varepsilon}(b) \cap U \neq 0$.

Theorem 4.3: let $A$ be $O B A$ and $f$ a linear functional from $A$ into $\mathbb{C}$ such that $f(e)=1$, then the two following conditions are equivalent

1. $f(a)=i \alpha$ then $f\left(a^{n}\right)=-\alpha^{n}$

2. $f$ is Character

Proof:- $(1) \rightarrow(2)$ To prove that $\mathrm{f}$ is character we have to prove that $f(a b)=f(a) f(b)$

if $f(a)=\alpha+i \beta$, then $f(a-\alpha)=i \beta$, by $(1)$

$f\left(a^{n}\right)=-\alpha^{n}$ $f\left((a-\alpha)^{n}\right)=-\beta^{n}$, then $(f(a-\alpha))^{n}=$

$(i \beta)^{n}=-\beta^{n}$

if $n=2 k+1, k=0,2,4, \ldots$

that is $f\left((a-\alpha)^{n}\right)=(f(a-\alpha))^{n}$

$\sum_{n=0}^{\infty}(-1)^{n}\left(\begin{array}{l}n \\ k\end{array}\right) f\left(a^{n-k}\right) \alpha^{k}=$ $\sum_{n=0}^{\infty}(-1)^{n}\left(\begin{array}{l}n \\ k\end{array}\right) f(a)^{n-k} \alpha^{k}$, then $f\left(a^{n-k}\right)=$ $f(a)^{n-k}$

so $f\left(a^{k+1}\right)=f(a)^{k+1}$ that is $f\left(a^{m}\right)=f(a)^{m}$ such that $m=k+1$, then $f$ is character

$(2) \rightarrow(1)$ since $f(a)=i \alpha$, then $f(a b)=$ $i \alpha f(b)$ for all $a, b \in A, \alpha \in \mathbb{R}$

since $f(a b)=f(a) f(b)$, then $f(a . a)=$ $f(a) \cdot f(a)$

then $f\left(a^{n}\right)=f(a)^{n}$ since $f(a)=i \alpha$ we have $(f(a))^{n}=(i \alpha)^{n}=$ $i^{n} \alpha^{n}=-\alpha^{n}$ such that $n=2 k+1, k=0,2,4, \ldots$

Theorem 4.4: let $A$ be a complex $O B A$ with identity and let $f$ be a character functional on $A$

$i$ - If $f(1)=0$, then $\|f\| \leq \varepsilon, 0<\varepsilon<1$

ii- If $f(1) \neq 0$, then $f(a) \in \mathcal{S} \mathcal{P}_{\epsilon}(a)$, for every $a \in A$, where $\varepsilon=\frac{\varepsilon^{\prime}}{\|f(1)\|}$

Proof:- (i) let $a, b \in A$, since $f$ is character. Then $f(a b)=f(a) f(b) \quad$ so $\quad f(a b)-f(a) f(b)=0$ if $b=1$, we obtain $\|f(a)\|=0<\varepsilon$, then $\|f\|<\varepsilon$.

(ii) suppose $f(a)=\beta$ and $\beta-a$ is invertible since $f$ is character then $f$ is continuous then $\left\|f(\beta-a) f(\beta-a)^{-1}\right\|=\| f((\beta-$ a) $(\beta-a)^{-1} \| \leq \varepsilon$ such that $\|\beta-a\| \|(\beta-$ $a)^{-1}\|\| \geq \frac{\|f(1)\|}{\varepsilon}$, then $\beta \in \mathcal{S} \mathcal{P}_{\epsilon}(a)$ 


\section{Definition 4.5(condition pseudo spectrum): Let}

$A$ be $O B A$ and $a \in A, \lambda \in \mathbb{C}$. The condition pseudo spectrum of $a$ which denoted by $\mathcal{P} \mathcal{S} \mathcal{P}_{\varepsilon}(a)$ is define by $\mathcal{P S}_{\mathcal{E}}(a)=\left\{\mathcal{S P}_{\varepsilon}(a) \cap\{\lambda \in \mathbb{C}:|\lambda|=\right.$ $\left.r_{\varepsilon}(a)\right\}$ for all $\lambda \in \mathbb{C}$.

In the following theorem we prove that the condition radius is convergent

Theorem 4.6: Let $A$ be a real $O B A$ and $\left\{a_{n}\right\}$ be a sequence, such that $a_{n} \rightarrow a$ as $n \rightarrow$ $\infty$.If $\mathcal{P} S \mathcal{P}_{\varepsilon}(a)$ contains at least one isolated point in $\delta \mathcal{P}_{\varepsilon}(a)$ then $r_{\varepsilon}\left(a_{n}\right) \rightarrow r_{\varepsilon}(a)$ as $n \rightarrow \infty$.

Proof:-Let $\lambda \epsilon \mathcal{P S P}_{\varepsilon}(a)$ isolated point in $\mathcal{S} \mathcal{P}_{\varepsilon}(a)$ and let $\mathcal{B}(\lambda, r)$ open set such that $\mathcal{B}(\lambda, r) \cap$ $\delta \mathcal{P}_{\varepsilon}(a)=\lambda$

let $V$ open set such that $\mathcal{B}(\lambda, r) \cap V=\emptyset$ that mean $\lambda \notin V$ and $\delta \mathcal{P}_{\varepsilon}(a) \backslash \lambda \subset V$ let $0 \leq k \leq r$. Then $\operatorname{sp}_{\varepsilon}(a) \subset$ $\mathcal{B}\left(k, r_{\varepsilon}(a)\right)$ from the upper semi continuous of the spectral radius and the fact $a_{n} \rightarrow a$ it follows that there exists $\mathcal{N}_{k_{1}}, \mathcal{N}_{k_{1}} \in \mathbb{N}$ such that $r_{\varepsilon}\left(a_{n}\right) \leq$ $r_{\varepsilon}(a)+k$ and $r_{\varepsilon}\left(a_{n}\right) \geq r_{\varepsilon}(a)-k$ for all $n \in \mathbb{N}$, we have $\delta \mathcal{P}_{\varepsilon}(a) \subset \mathcal{B}(\lambda, k) \cup V$ and $\mathcal{S} \mathcal{P}_{\varepsilon}(a) \cap$ $\mathcal{B}(\lambda, k) \neq \varnothing$ so by using theorem(4.4.2[9]) $\mathcal{S} \mathcal{P}_{\varepsilon}\left(a_{n}\right) \cap \mathcal{B}(\lambda, k) \neq \emptyset$ suppose $\alpha_{n} \in \mathcal{S} \mathcal{P}_{\varepsilon}(a)$ and $\left|\alpha_{n}-\lambda\right|<k_{1}$ for all $n \geq \mathcal{N}_{k_{1}}$, then $r_{\varepsilon}(a)$ $\geq\left|\alpha_{n}\right|>r_{\varepsilon}(a)-\varepsilon$ let $\mathcal{N}=\max \left\{\mathcal{N}_{k_{1}}, \mathcal{N}_{k_{2}}\right\}$, so $r_{\varepsilon}(a)-k<$ $r_{\varepsilon}\left(a_{n}\right)<r_{\varepsilon}(a)+k$ that mean $-k<r_{\varepsilon}\left(a_{n}\right)-$ $r_{\varepsilon}(a)<k$ $\left|r_{\varepsilon}\left(a_{n}\right)-r_{\varepsilon}(a) \quad\right|<k$ that $\operatorname{mean} r_{\varepsilon}\left(a_{n}\right) \rightarrow$ $r_{\varepsilon}(a)$

$\square$

Definition 4.7: let $A$ be complex $O B A$ with identity, we define the distance between $\lambda \in \mathbb{C}$ and the $\mathcal{S} \mathcal{P}_{\varepsilon}(a)$ by

$$
d\left(\lambda, \mathcal{S} \mathcal{P}_{\varepsilon}(a)\right)=\inf \left\{|\lambda-\beta|: \beta \in \mathcal{S} \mathcal{P}_{\varepsilon}(a)\right\} \text {. And }
$$
if $\lambda=0$ the distance was between 0 and $\delta \mathcal{P}_{\varepsilon}(a)$ will denoted by $\delta(a)=d\left(0, \delta \mathcal{P}_{\varepsilon}(a)\right)$

Theorem 4.8: let $A$ be $O B A$ and $C$ is closed normal and closed-inverse algebra cone, if $a \in A$,then $\delta(a) \in \mathcal{S} \mathcal{P}_{\varepsilon}(a)$.

Proof:- if $a \notin I N(a)$, then $\delta(a)=0 \epsilon \mathcal{S} \mathcal{P}_{\varepsilon}(a)$ suppose that $a \in I N(a)$, since $a \in C$ and $C$ is inverseclosed then $a^{-1} \epsilon C$ since $C$ is normal and closed then $r_{\varepsilon}\left(a^{-1}\right) \epsilon \mathcal{S} \mathcal{P}_{\varepsilon}\left(a^{-1}\right)$

so that $r_{\varepsilon}\left(a^{-1}\right)=\frac{1}{\alpha+i t}$, for some $\alpha+i t \in \mathcal{S} \mathcal{P}_{\varepsilon}(a)$ since $r_{\varepsilon}\left(a^{-1}\right)=\frac{1}{\delta(a)}$, then by comparing with $(*)$ obtain $\delta(a) \epsilon \mathcal{S} \mathcal{P}_{\mathcal{\varepsilon}}(a)$. 


\section{references}

[1] D.Holland, "Banach Algebra Notes", Oregon State University, American, (2015).

[2] D. Sukumar, "Ransford Spectrum in Banach Algebra", Ph. D. Thesis India Institute of Technology Madras, India, January (2007).

[5] H. Raubenheimer and S. Rode" Cone in Banach Algebra", Ms. thesis, University of the Orange Free State, India Mayhem, .N.S 7(4). pp.489.502, (1996).

[6] K. Muzundu, "Spectral Theory in Commutative Order Banach Algebras", Ph.D. Thesis, Stellenbosch University, (2012).

[7] K. Yosida, "On the Theory of Spectra ", Osaka Imperial University,(1940).
[3] E. B. Daies,"Spectral Theory and Differential Operators", Cambridge University press,(1996).

[4] G.Corach and F. D. Suarez, "Extention of Characters in Commutative Banach Algebra ", Buenos Aires University, (1987).

[8] R. De Jong, "Ordered Banach Algebras", Ms. Thesis, Leiden University, (2010).

[9] Sarah F., "On Banach algebra", Ms. Thesis. Alqadisiya University, Iraq, (2017)

$$
\text { الطيف المرثب الثرطي }
$$

$$
\begin{aligned}
& \text { سارة فاهم حسن } \\
& \text { قسم الرياضيات } \\
& \text { كلية علوم الحاسوب وتكنلوجيا المعلومات } \\
& \text { جامعة القادسية ولموبة }
\end{aligned}
$$

Sarafahim66@yahoo.com

$$
\text { بشرى يوسف حسين ال رياضية التربت }
$$

Boushra.alshebani@qu.edu.iq

\section{: - المستخلص :}

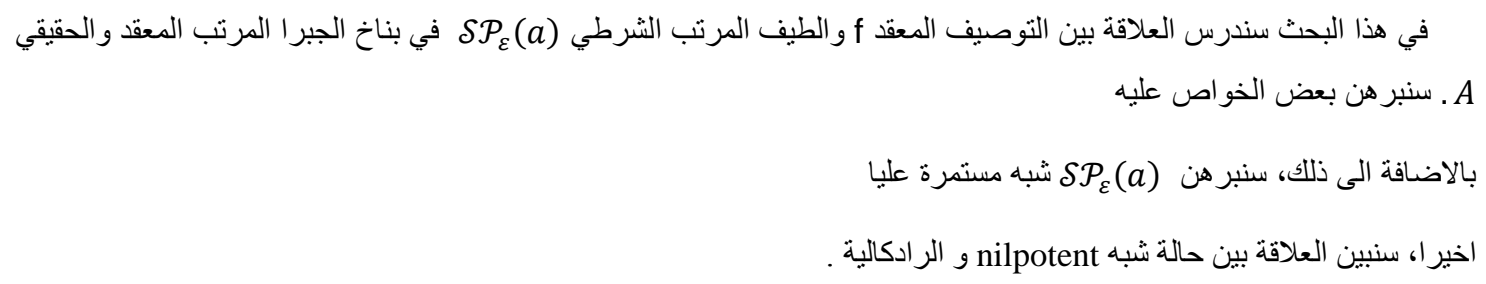

